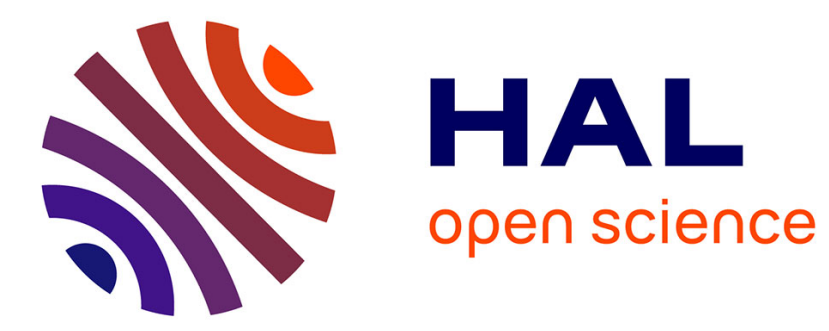

\title{
Antarctique et espace : fin et suite de la géographie
}

Sebastian V. Grevsmühl

\section{To cite this version:}

Sebastian V. Grevsmühl. Antarctique et espace : fin et suite de la géographie. L'Information géographique, 2010, 74 (2), pp.115. 10.3917/lig.742.0115 . hal-01502178

\section{HAL Id: hal-01502178 \\ https://hal.science/hal-01502178}

Submitted on 7 Apr 2017

HAL is a multi-disciplinary open access archive for the deposit and dissemination of scientific research documents, whether they are published or not. The documents may come from teaching and research institutions in France or abroad, or from public or private research centers.
L'archive ouverte pluridisciplinaire HAL, est destinée au dépôt et à la diffusion de documents scientifiques de niveau recherche, publiés ou non, émanant des établissements d'enseignement et de recherche français ou étrangers, des laboratoires publics ou privés. 


\title{
Antarctique et espace : fin et suite de la géographie
}

\author{
par Sebastian V. Grevsmühl
}

Sebastian V. Grevsmühl, histoire des sciences, Centre Alexandre Koyré, CNRS/ EHESS

grevsmuehl@damesme.cnrs.fr

\section{L'Antarctique et l'Espace}

Les régions polaires, et l'Antarctique en particulier, à cause de son isolement géographique extrême, entretiennent une relation particulière avec l'exploration spatiale. Des analogies avec l'Espace tel qu'on se le représentait se sont déjà imposées aux premiers explorateurs qui ont pénétré cette région isolée et hostile. Lors de la grande expédition polaire britannique, l'expédition Discovery (1901-1904) qui suivit celle de James Clark Ross, Edward Wilson a consigné dans son journal, le 22 mai 1902 : « Le silence était presque inquiétant. On pouvait s'imaginer sur une autre planète, morte. Je pouvais facilement imaginer que nous n'étions pas sur la Terre, mais sur la surface de la Lune. Tout était tellement calme et mort et froid et loin du monde terrestre ${ }^{1}$. » Cette expérience radicalement nouvelle fut partagée par beaucoup d'autres explorateurs courageux, notamment par l'aviateur et explorateur américain, l'amiral Richard Byrd, qui a écrit en 1938 dans son journal Alone : « Je me sentais comme si j'étais tombé sur une autre planète ou dans un autre horizon géologique dont l'homme ne possédait ni connaissance ni mémoire ${ }^{2}$.»Visiblement, cette région possède des caractéristiques uniques qui ne laissent personne indifférent.

La spécificité de l'Antarctique par rapport aux autres régions polaires tient au fait que c'est un continent entièrement entouré d'océans alors que l'Arctique est une mer (gelée) entourée de sol et donc d'États souverains. En conséquence, on peut observer en Arctique une histoire riche en inte-

1. Cité d'après Desmond J. Lugg, "Antarctica as Space Laboratory », in Gotthilf Hempel (éd.), Antarctic Science, Global Concerns, Berlin, Heidelberg, New York, Springer Verlag, 1994, p. 229, traduit par l'auteur.

2. Richard Byrd, Alone, The Classic Polar Adventure, New York, Kodansha, 2003 [1938], p. 120, trad. par l'auteur. 


\section{Antarctique et espace : fin et suite de la géographie}

ractions avec les peuples indigènes et les pays riverains aussi bien au niveau géopolitique, qu'au niveau économique, culturel et social. L'Antarctique, en revanche, est très loin de cette situation. Ne possédant pas de peuple indigène, le continent antarctique ne connaît qu'une version extrêmement simplifiée d'une histoire culturelle et humaine. Gouverné de fait aujourd'hui par les membres du traité sur l'Antarctique (voir encadré sur le droit de l'espace p. 127) qui ont un «statut consultatif », les parties colonisées de l'Antarctique réclamées par sept pays ne bénéficient de reconnaissance ni par la communauté mondiale ni par le droit international. En fait, l'Antarctique est restée pratiquement impénétrable jusqu'à la fin $\mathrm{du} \mathrm{XIX}^{\mathrm{e}}$ siècle et c'est seulement pendant la deuxième moitié $\mathrm{du}$ $\mathrm{XX}^{\mathrm{e}}$ siècle qu'elle est devenue un lieu habituel de pratique des sciences. Autrement dit, c'est un espace qui se trouve à tous les niveaux à la périphérie mondiale, car il s'agit d'un véritable «pôle à part ${ }^{3}$ » aussi bien à cause de sa localisation périphérique qu'à cause de son isolement géographique et de son environnement radicalement hostile à la présence humaine du fait de conditions météorologiques extrêmes. Si on cherche sur notre planète un lieu qui convienne à ce qu'on pourrait qualifier « une expérience spatiale », l'Antarctique est a priori le seul candidat sérieux même si certaines conditions (gravité, amplitude thermique, éloignement, moyens d'accès etc.) ne sont bien sûr pas ou très mal représentées.

\section{La fin de la géographie terrestre}

Ce caractère de modèle relève d'un développement particulier dans l'histoire de l'exploration dans laquelle l'Antarctique figure comme région clé. Lors du sixième Congrès International de Géographie qui s'est tenu en 1895 à Londres, le fameux océanographe canadien John Murray a constaté que « l'exploration des régions antarctiques est la plus grande affaire de l'exploration géographique encore à entreprendre ${ }^{4} \gg$. Ce constat ouvre la période que l'on identifie aujourd'hui comme «l'âge héroïque » de l'exploration antarctique, et qui a vu son apogée avant tout dans la course au pôle Sud au début du $\mathrm{XX}^{\mathrm{e}}$ siècle. Depuis ce temps, on se réfère fréquemment à l'Antarctique comme « la dernière frontière » ou bien «le dernier lieu », évoquant non seulement le vieux thème de la terra incognita, mais surtout le fait (qui peut sembler tout d'abord trivial) que l'Antarctique fut le tout dernier conti-

3. Philip W. Quigg, A Pole Apart, The Emerging Issue of Antarctica, New York, New Press, 1983.

4. Voir par exemple : Walter Sullivan, Quest for a Continent, Londres, Secker \& Warburg, 1957, p. 35 ; Laurence P. Kirwan, A History of Polar Exploration, New York, W.W. Norton, 1959, p. 224 et Sanjay Chaturvedi, The Polar Regions : A Political Geography, Chichester, New York, J. Wiley, 1996, p. 63. 
nent exploré et la toute dernière tranche de sol dont les États aient pu prendre possession. Ce simple fait géographique a néanmoins entraîné de profondes conséquences, car avec l'ouverture de l'exploration du continent antarctique, tout un âge d'exploration - et en conséquence toute une ère de découverte géographique - se sont achevés.

Un des penseurs les plus rigoureux (et le plus controversé) de la thèse de la fin de la géographie fut probablement le géographe et politicien britannique Halford John Mackinder, souvent considéré comme le père fondateur de la théorie géopolitique. En 1904, lors d'un discours présenté devant la Royal Geographical Society, Halford Mackinder a formulé une importante thèse qui est devenue depuis un classique ${ }^{5}$. Son «hypothèse d'un espace clos » repose sur la vision d'un globe complètement fermé, un monde dans lequel toutes les activités, comme elles sont limitées à une terre pratiquement entièrement explorée, sont en interaction les unes avec les autres - une vision qui peut d'ailleurs facilement être rapprochée (sous d'autres prémisses évidemment) de la fameuse métaphore de Buckminster Fuller du «Vaisseau Terre ${ }^{6} »$ Le monde, selon Halford Mackinder, est devenu un globe unifié de lieux occupés et des événements qui se produisent dans une partie du monde provoquent inévitablement des conséquences dans d'autres parties. Sans discuter ici les aspects problématiques de l'ethnocentrisme de cette thèse et son usage historique dans des discours raciaux, l'émergence de cette hypothèse tout au début du $\mathrm{XX}^{\mathrm{e}}$ siècle est avant tout un indice important du changement profond de la perception de l'espace en Occident. L'Antarctique occupe évidemment une place décisive dans cette interprétation de l'histoire et de la pensée spatiale occidentales.

L'idée de rupture, c'est-à-dire d'une histoire non-linéaire qui ne se laisse justement plus décrire d'une façon chronologique - idée qui est déjà très présente chez Halford Mackinder -, a été reprise dans d'autres approches plus récentes, notamment dans l'histoire de l'exploration New Lands, New Men : America and the Second Great Age of Discovery. Selon l'historien américain William Goetzmann, le XVIII ${ }^{\mathrm{e}}$ siècle a vu émerger une nouvelle forme d'exploration, ce qu'il appelle le «deuxième grand âge de découverte», avec les voyages du capitaine Cook et d'Alexander von Humboldt qui ouvraient une nouvelle ère d'exploration géographique stimulée par la révolution scientifique ${ }^{7}$. En histoire environnementale, Stephen Pyne a repris cette périodisation en y ajoutant une troisième période. Selon lui, tandis que

5. Voir Halford John Mackinder, "The Geographical Pivot of History », The Geographical Journal, vol. 23, n 4, 1904, p. 421-437.

6. Pour une bonne introduction à la pensée de H. Mackinder, voir surtout le premier chapitre dans : Gearóid Ó Tuathail, Critical Geopolitics, The Politics of Writing Global Space, Londres, Routledge, 1996.

7. William Goetzmann, New Lands, New Men : America and the Second Great Age of Discovery, New York, Viking, 1986. 
la première période qui s'étend du $\mathrm{XV}^{\mathrm{e}}$ au $\mathrm{XVII}^{\mathrm{e}}$ siècle a été surtout marquée par les explorations des mers, le « second âge de la découverte » se caractérise avant tout par l'exploration des continents qui constituait une occupation majeure des XVIII ${ }^{\mathrm{e}}$ et XIX ${ }^{\mathrm{e}}$ siècles. Ce sont les traversées transcontinentales qui représentaient des voyages paradigmatiques de cette époque. Quand, par exemple en 1914, le Britannique Ernest Shackleton tente son fameux voyage TransAntarctique, c'est en quelque sorte la tentative d'accomplissement de la toute dernière étape d'un projet qui était déjà achevé pour tous les autres continents. Dans ce sens, l'atteinte du pôle Sud par Roald Amundsen et ses compagnons en 1911 annonce déjà non seulement la fin de cette deuxième période d'exploration, mais surtout la fin de toute une ère géographique. L'Antarctique a clairement figuré comme dernier lieu sur cette liste des lieux d'explorations, le tout dernier d'un monde qui est devenu entièrement clos et qui s'est vite vu dépassé par un nouvel âge d'exploration - qui correspond à la troisième période de Pyne - avec l'émergence d'une nouvelle frontière, celle de l'Espace. Autrement dit, avec l'accomplissement des grands voyages antarctiques, on a assisté à la fin de la géographie terrestre ${ }^{8}$. Et c'est justement la fin de la géographie terrestre qui a permis sur le plan historique de tisser des liens forts entre l'Antarctique et l'Espace en proposant une ouverture à de nouvelles géographies.

\section{Laboratoires terrestres et spatiaux}

L'émergence de différentes utilisations de la notion de laboratoire liées aux pratiques et imaginaires antarctiques montre bien la dimension de modèle de ce milieu particulier. D'une façon très générale, on fait souvent référence à l'Antarctique comme "au plus grand laboratoire du monde », car l'Antarctique offre en effet un espace expérimental extrêmement divers. Les sciences naturelles ont tendance à souligner le caractère pur et immaculé de l'espace antarctique et parlent en conséquence du plus grand « laboratoire naturel »du monde, en pointant justement des conditions physiques spécifiques qu'on peut trouver uniquement au-delà de la latitude du $60^{\circ} \mathrm{Sud}^{9}$. Cette métaphore fut tout d'abord introduite en 1779 par le naturaliste suisse Horace-Bénédict de Saussure avec la formule de « laboratoire de la nature », désignant des phénomènes qu'on ne peut trouver que dans les milieux alpins. Un siècle plus tard, cette métaphore est devenue banale et on voit sa diffusion dans beaucoup de

8. Stephen Pyne, «The extraterrestrial Earth : Antarctica as analogue for space exploration », Space Policy, vol. 23, n³, 2007, p. 147-149; Stephen Pyne, "Seeking Newer Worlds: An Historical Context for Space Exploration », in Steve Dick, Roger Launius (éd.), Critical Issues in the History of Spaceflight, Washington DC, NASA, 2006, p. 7-35.

9. Il existe plusieurs définitions pour la «région antarctique»; nous avons choisi d'adopter ici la définition de l'article IV du traité sur l'Antarctique. 
domaines de sciences naturelles ${ }^{10}$. Désormais, au lieu de faire entrer la nature dans le laboratoire, on préfère déplacer le laboratoire dans la nature. L'idée que des conditions physiques - qu'on sait plus ou moins reproduire dans des laboratoires - se laissent davantage observer dans leur milieu naturel, trouve de nombreuses nouvelles applications au cours du $\mathrm{XX}^{\mathrm{e}}$ siècle. Dans ce sens, dès leur découverte, ces environnements des extrêmes - qu'ils soient montagneux, polaires ou spatiaux - sont perçus comme offrant à la fois un défi et une opportunité ; un défi à cause des efforts physiques, mentaux, logistiques ou économiques qu'ils exigent des visiteurs et une opportunité scientifique à cause des conditions physiques particulières qu'on y trouve. La persistance historique de la métaphore du «laboratoire naturel» est en même temps un indice important de la régression constante de la distinction classique entre les sciences de terrain et les sciences de laboratoire. L'Antarctique - et cela compte en partie aussi pour l'Espace - est dans ce sens, d'une façon paradoxale, tout à la fois : lieu d'étude et objet à étudier.

Au cours de l'histoire (dans le cas de l'Antarctique depuis l'Année Géophysique Internationale de 1957-1958), ces défis sont eux-mêmes entrés dans le domaine des sciences et les métaphores de laboratoire se sont rapidement multipliées. L'espace antarctique offre aux sciences de la vie au sens large, c'est-à-dire aux psychologues, aux éthologues et aux biologistes humains, une chance unique pour étudier l'homme dans ce qu'ils appellent un «laboratoire humain». Les études sur les effets divers du confinement extrême sur la psyché, ainsi que l'observation des interactions sociales complexes dans un environnement hostile et confiné sont devenues entre-temps des études polaires classiques qui ne manquent pas d'inspirer le domaine de l'exploration spatiale ${ }^{11}$. Les récits et écrits de grands explorateurs comme de l'Australien Douglas Mawson ou de l'amiral américain Richard Byrd évoqués déjà plus haut constituent d'ailleurs les premières sources importantes de ce type de témoignage de défis psychologiques extraordinaires - expérience qu'on pourrait nommer «l'expérience antarctique ${ }^{12} »$.

10. Pour une discussion de cette métaphore, voir le numéro spécial : Charlotte Bigg, David Aubin, Philipp Felsch, "Introduction: The Laboratory of Nature - Science in the Mountains », Science in Context, vol. 22, $\mathrm{n}^{\circ} 3,2009$, p. 311-321.

11. Voir par exemple : Albert A. Harrison, Yvonne A. Clearwater, Christopher P. McKay (éd.), From Antarctica to Outer Space, Life in Isolation and Confinement, New York, Springer Verlag, 1991 ; Jack Stuster, Bold Endeavors, Lessons from Polar and Space Exploration, Annapolis, Naval Institute Press, 1996 ; Peter Suedfeld, Karine Weiss, « Antarctica : Natural Laboratory and Space Analogue for Psychological Research », Environment and Behavior, vol. 32, $\mathrm{n}^{\circ} 1$, 2000, p. 7-17; Carole Tafforin, "Ethological Analysis of a Polar Team in the French Antarctic Station Dumont d'Urville as Simulation of Space Teams for Future Interplanetary Missions », Acta Astronautica, vol. 55, n 1, 2004, p. 51-60.

12. On peut avoir une très bonne idée de ces défis multiples en lisant les extraits des récits et écrits des grands explorateurs rassemblés par exemple dans : Charles Neider (éd.), Antartica, Authentic Accounts of Life and Exploration in the World's Highest, Driest, Windiest, Coldest and Most Remote Continent, New York, Dorsett Press, 1972. 


\section{Antarctique et espace : fin et suite de la géographie}

Ces études de l'impact environnemental sur l'homme dans des conditions physiques extrêmes, et qui avaient été menées par exemple au XIX $X^{e}$ siècle sous forme d'expériences en haute altitude dans les régions alpines et le milieu des aérostiers ${ }^{13}$, se sont étendues au $\mathrm{XX}^{\mathrm{e}}$ siècle aux autres régions difficilement accessibles dont notamment l'Antarctique qui figurait comme un lieu important d'application. Le pas vers le « laboratoire spatial» n'était pas loin.

Cette idée d'utiliser la région antarctique comme lieu d'introduction à l'exploration spatiale fut illustrée dans le cas des États-Unis par la visite pendant une semaine en janvier 1967 de sept bases antarctiques par quatre membres influents de la NASA. Le fameux Wernher von Braun, à l'époque directeur du Marshall Space Flight Center, était accompagné par Ernst Stuhlinger, directeur du Space Sciences Laboratory (du Marshall Space Flight Center), et de deux autres directeurs importants, Robert Gilruth et Maxime Faget du Manned Spacecraft Center à Houston. Le but de cette excursion - qui visait aussi à améliorer les relations entre von Braun et Gilruth - était d'identifier les leçons à tirer du programme de recherche antarctique américain pour des éventuelles applications dans le domaine des explorations habitées dans l'Espace, notamment pour une base lunaire ou martienne mobile (voir encadré p. 126). Les préoccupations fondamentales étaient de nature logistique et l'équipe s'est surtout montré intéressée par la gestion du personnel, des stations, des habitations et des systèmes d'entretien de la vie ainsi que par l'utilisation des terres incultes, notamment les vallées sèches, pour tester des instruments utilisés en études spatiales afin de détecter des signes de vie sur d'autres planètes ${ }^{14}$.

Les leçons tirées de cette expérience furent notamment la nécessité de maintenir ce qu'on appelle "le facteur ou l'élément humain ${ }^{15}$ », c'est-à-dire le fait d'envoyer en personne des scientifiques sur le terrain pour faire des observations in situ. Même si la NASA a affiché cette position jusqu'à aujourd'hui, dans la pratique, le géologue américain Harrison Schmitt fut le

13. Pour les expériences en haute altitude, voir par exemple: Philipp Felsch, « Mountains of Sublimity, Mountains of Fatigue : Towards a History of Speechlessness in the Alps », Science in Context, vol. 22, $\mathrm{n}^{\circ}$ 3, 2009, p. 341-364; pour le milieu des aérostiers en France, voir par exemple : Fabien Locher, « De nouveaux territoires pour les sciences: Les voyages aériens de Camille Flammarion », Sociétés et Représentations, vol. 1, n²1, 2006, p. 157-173.

14. Ernst Stuhlinger, "Antarctic Research, A Prelude to Space Research », Antarctic Journal of the United States, vol. 4, $\mathrm{n}^{\circ}$ 1, 1969, p. 1-7 ; Wernher von Braun, «A Space Man's Look at Antarctica », Popular Science, mai 1967, p. 114-116,200; Michael J. Neufeld, Von Braun : Dreamer of Space, Engineer of War, New York, Knopf, 2007 ; Tillman Durdin, « Antarctic Studies Said to Aid US Space Program Research », New York Times, 15 janvier 1967, p. 87.

15. Dans le monde anglophone, il existe une littérature abondante sur le « human element » dans le vol spatial. Pour une bonne introduction au domaine et sur les tensions et alternatives entre l'élément humain et les machines, voir "Part II: Human and Robotic Exploration » dans : Steve Dick, Roger Launius (éd.), Critical Issues in the History of Spaceflight, Washington DC, NASA, 2006. 
premier, mais aussi le dernier, scientifique à être envoyé sur la Lune et ce lors du dernier voyage pendant la mission Apollo-17 en 1972. Peut-être plus importants encore, les courts récits enthousiastes auxquels cette visite en Antarctique a donné lieu montrent l'émergence de tous les thèmes principaux qui vont se retrouver dans les différents programmes de recherche actuellement mis en œuvre par plusieurs États membres du Traité sur l'Antarctique. Le développement probablement le plus important dans ce cadre fut la création de la «Working Group on Human Biology and Medicine », en septembre 1974, sous le toit du SCAR (Scientific Committee on Antarctic Research).

Ainsi, une étude de 1971 du National Research Council résumant les expériences qui ont porté pendant les années 1960 sur le facteur humain, conclut que « la base américaine au pôle Sud est un site idéal pour une large gamme d'expériences en recherche fondamentale portant sur l'adaptation humaine, l'interaction homme-machine et la dynamique de groupes de petite taille ${ }^{16} »$. Grâce à l'établissement de la "Working Group on Human Biology and Medicine », ainsi que les multiples efforts personnels d'Eric Gunderson, d'A.G. Owens et de Jacques Rivolier ${ }^{17}$, les sciences comportementales et psychologiques se sont durablement établies en Antarctique pendant la période d'après-guerre et l'exploration spatiale a pu largement profiter de ces développements.

\section{L'analogie antarctique}

Un dernier élément qui mérite une considération plus particulière est le cadre juridique qui a également joué un rôle fondamental dans le développement de ces approches par analogie. Au niveau juridique, de nouvelles conventions ont dû être négociées pour réglementer à une échelle internationale ces conquêtes ambitieuses. Cela se reflète dans le domaine du droit international public avec l'émergence pendant les vingt premières années suivant la deuxième Guerre mondiale de nombreux nouveaux traités portant notamment sur le droit de la mer (United Nations Convention on the Law of the

16. National Research Council, Biomedical and Behavioral Science Research in Antarctica, Review and Recommendations, Washington DC, NAS/NRC, 1971, p. 3-4, traduit par l'auteur.

17. Voir par exemple: Eric Gunderson, «Emotional symptoms in extremely isolated groups », Archives of General Psychiatry, vol. 9, n 4, 1963, p. 362-368; Eric Gunderson (éd.), Human adaptability to Antarctic conditions, Antarctic research series, vol. 22, Washington DC, American Geophysical Union, 1974 ; A.G. Owens, « Some biographical correlates of assessed performance in small Antarctic groups », Australian Army, Psychological Research Unit, Research report, $\mathrm{n}^{\circ}$ 12-68, 1968 ; Jacques Rivolier et al., « Crew selection for an antarctic-based space simulator », in Albert A. Harrison, Yvonne A. Clearwater, Christopher P. McKay (éd.), From Antarctica to Outer Space, Life in Isolation and Confinement, New York, Springer Verlag, 1991 , p. 291-296. 


\section{Antarctique et espace : fin et suite de la géographie}

Sea, UNCLOS I-III, dont les premières conférences se sont tenues entre 1956-1958) et le droit de l'espace (1967). Ce développement historique unique ne peut être pas compris sans prendre en compte la naissance du Traité sur l'Antarctique en 1959 et son évolution constante d'une régulation relativement simple à un véritable système de référence juridique d'une grande complexité. C'est dans cette évolution historique que le droit international public a inventé la notion de «l'analogie antarctique ${ }^{18}$ » dans son sens juridique.

Le terme et le concept d'analogie antarctique ont été développés et appliqués peu après le Traité sur l'Antarctique (1959) dans le cadre du Traité de l'Espace de 1967 et les négociations sur le Traité de la Lune pendant les années 1970.

De nombreux juristes ont déjà souligné l'importance de l'analogie antarctique comme outil permettant de formuler des régulations internationales pour l'Espace ${ }^{19}$. Ainsi, le souvenir du succès du Traité sur l'Antarctique était toujours très présent dans les cercles diplomatiques pendant la deuxième moitié des années 1960 et il a permis à son architecte principal, les États-Unis, d'introduire une bonne partie de leurs propositions dans les négociations spatiales.

Dit d'une façon très brève, d'après la juriste Peterson, le Traité sur l'Antarctique a servi surtout de modèle pour réunir à la fois la non-appropriation territoriale et la conservation de la souveraineté nationale sur le matériel et les scientifiques envoyés. De la même manière que l'analogie de la haute mer a inspiré le concept d'État du pavillon (flag state jurisdiction), c'est-à-dire la réclamation légale extraterritoriale de véhicules dans l'Espace, l'analogie antarctique a permis d'adapter les règles de souveraineté nationale du Traité sur l'Antarctique aux cas de missions scientifiques et de construction de bases de recherche dans l'Espace.

D'autres aspects juridiques importants ont été discutés par la suite dans les cercles diplomatiques surtout pendant les années 1970, notamment la question des ressources de minéraux ${ }^{20}$, question qui ne fut évoquée ni dans le Traité sur l'Antarctique, ni dans le Traité de l'Espace et qui a joué un rôle décisif dans l'émergence de ce qu'on pourrait appeler un «régime environnemental » en Antarctique. Dit d'une façon beaucoup plus générale, des concepts comme le patrimoine commun de l'humanité, la notion de world park proposée pour l'Antarctique en 1972 par Greenpeace ou bien la notion de réserve naturelle ont tous évolué et changé considérablement pen-

18. Le terme vient de l'étude pionnière de Philip Jessup, Howard Taubenfeld, Controls for Outer Space and the Antarctic Analogy, New York, Columbia University Press, 1959.

19. Une des meilleures études juridiques portant sur l'analogie antarctique et celle de la juriste M. J. Peterson, "The use of analogies in developing outer space law », International Organization, vol. 51, $\mathrm{n}^{\circ} 2$, 1997, p. 245-274.

20. Pour une bonne introduction à cette question voir : Elisabeth Mann Borgese, The Mines of Neptune, Minerals and Metals from the Sea, New York, Abrams, 1985. 
dant la période d'après Guerre dans le triangle conceptuel entre mer, Espace et Antarctique ${ }^{21}$. Il reste à voir si l'analogie antarctique jouera le même rôle dans les futures négociations sur l'exploitation des ressources naturelles spatiales quand le moment en sera venu.

\section{- Voyages imaginaires et retour à la Terre}

Malgré les multiples efforts scientifiques pour faire de l'Antarctique un lieu pouvant servir à accéder à d'autres espaces et à d'autres mondes, notamment l'espace extra-terrestre, les relations les plus étroites entre l'Antarctique et l'Espace relèvent probablement d'un registre symbolique et imaginaire. En tant que lieu profondément abiotique et même «a-culturel», marqué par l'absence complète de tout ordre social et de toute histoire indigène, le septième continent propose avant tout un écran idéal pour des projections d'imaginaires.

Dans cette logique, une histoire de l'analogie antarctique devrait incorporer aussi le champ de la littérature, car l'Antarctique, et le pôle Sud en particulier, furent imaginés, dès l'émergence de leur adaptation littéraire, comme passerelles vers de nouvelles topographies et de nouveaux mondes. Deux types de récits semblent dominer ce champ. Des voyages vers l'intérieur de la Terre sont le thème principal de nombreuses utopies. «Symzonia : A Voyage of Discovery » de 1820 dans laquelle le pôle Sud apparaît comme un portail qui permet l'accès à l'intérieur de la Terre est certainement l'histoire la plus connue. Dans un deuxième type de fiction on retrouve le leitmotiv de notre article, avec une mise en scène du pôle Sud comme lieu de passage vers l'Espace, comme notamment dans «Armata : A Fragment» (1816 et 1817) de Thomas Erskine et «Journey to Mars » (1894) de Gustavus W. Pope ${ }^{22}$. Ces utopies et fictions furent néanmoins largement inspirées par des récits et journaux de voyages bien réels et le champ de la fiction antarctique a largement évolué en lien étroit avec les grands voyages exploratoires ${ }^{23}$.

21. Pour une discussion approfondie de l'évolution du Traité sur l'Antarctique, voir: Arthur Watts, International Law and the Antarctic Treaty System, Cambridge, Grotius, 1992 ; pour des bonnes introductions et une présentation du concept de "world park » on se réfère à M. J. Peterson, Managing the Frozen South, Berkeley, Los Angeles, Londres, University of California Press, 1988, p. 118 et Christopher Joyner, Sudir Chopra, The Antarctic Legal Regime, Boston, Londres, Martinus Nijhoff Publishers, 1988, p. 38-39.

22. Captain Adam Seaborn [pseudonyme], Symzonia : Voyage of Discovery, New York, J. Seymour, 1820 ; Thomas Erskine, Armata : A fragment, London : John Murray, Part I, 1816, Part II, 1817 ; Gustavus W. Pope, Journey to Mars, New York, G. W. Dillingham, 1894.

23. Voir Elizabeth Leane, "Romancing the Pole : A Survey of Nineteenth Century Utopias », $A C H, n^{\circ} 23,2004$, p. 147-171; voir aussi : Frédérique Rémy, L'Histoire des pôles, mythes et réalités polaires, XII et XVIII ${ }^{e}$ siècles, Paris, Desjonquères, 2009. 
Mais ce qui distingue finalement l'exploration de l'Antarctique et de l'Espace de celles des autres grands âges d'exploration est le fait qu'il n'y avait plus personne à rencontrer d'autre que soi-même. Le parallélisme entre l'Antarctique et l'Espace, que les sciences ont (ré)inventé au cours du $\mathrm{XX}^{\mathrm{e}}$ siècle, renvoie finalement à un geste profondément autoréférentiel qui caractérise non seulement l'exploration de l'Antarctique, mais aussi celle de l'Espace. Lors de notre nouvelle ère de voyages, l'homme est renvoyé à luimême. Les explorateurs de l'Arctique, par exemple, ont pu se nourrir de la faune locale, ils ont compté sur l'aptitude des conducteurs de traîneau indigènes et ils ont pu utiliser les technologies locales. Dans le cas de l'exploration de l'Antarctique, il n'y avait aucun savoir local à adapter car il n'y avait personne à rencontrer. En conséquence, les explorateurs de l'Antarctique, comme justement ceux de l'Espace, n'ont pas seulement dû tout amener avec eux-mêmes mais leurs voyages n'ont finalement fait que les renvoyer à leur propre existence.

Fig. 1 : Cette photo est la première qui a été prise de la Terre depuis la Lune avec un film noir et blanc. Ce seront les images couleur prises peu après qui deviendront iconiques. La position de la Terre par rapport à la Lune pour inhabituelle qu'elle soit correspond à l'orbite du vaisseau qui était située dans le plan équatorial lunaire.

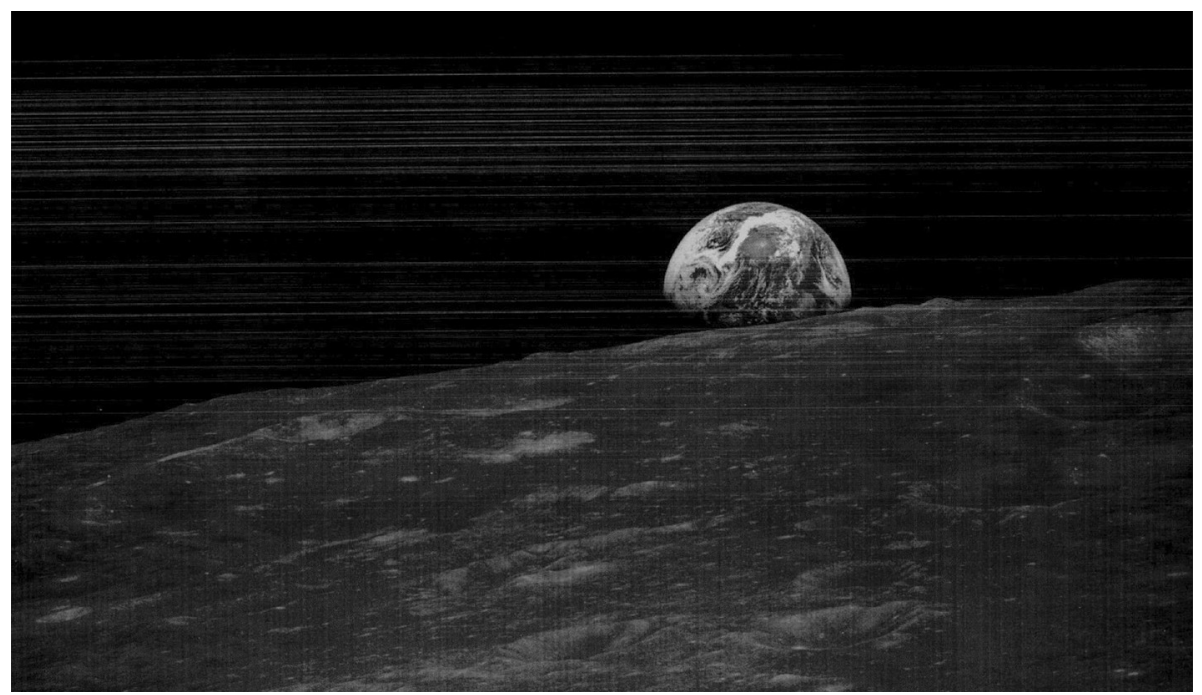

Source : http://history.nasa.gov/ap08fi/photos/e/as08-13-2329.jpg

Cet aspect autoréférentiel est désormais incontournable. Ce qu'un des philosophes les plus attentifs à ces nouvelles entreprises exploratoires, Günther Anders a écrit entre 1968 et 1969 sur les voyages à la Lune a été déjà anticipé en quelque sorte par les explorateurs solitaires du pôle Sud : « [...] l'événement décisif des voyages spatiaux ne réside pas dans le fait 
d'atteindre des régions lointaines de l'Espace [...], mais dans le fait que la Terre a pour la première fois la chance de se voir elle-même, de se rencontrer d'une manière identique à l'homme qui se rencontre en se reflétant dans un miroir ${ }^{24}$. » Les fameuses photographies de la Terre vue de l'espace qui ont été prises dans le cadre du programme Apollo et auxquelles Günther Anders fait référence, reflètent précisément ce caractère autoréférentiel. Une photographie en particulier, la fameuse «Earthrise » (« lever de Terre ») de 1968 - est devenue entre-temps iconique, car elle a profondément marqué le début d'une conscience environnementale globale en stimulant justement des contemplations sur notre propre existence et la place que nous occupons dans l'univers (fig. 1). Et c'est précisément seulement un an plus tard, lors du premier alunissage de l'homme en 1969, que le fondateur des Expéditions polaires françaises Paul-Émile Victor a dessiné le croquis présenté figure 2, qui illustre toute cette dialectique des regards dans laquelle l'explorateur seul face à lui-même, qu'il soit sur l'Antarctique ou sur la Lune, se découvre un double lointain.

Fig. 2 : Carte postale, croquis de Paul-Émile Victor, « L'homme en Antarctique félicite le premier Américain sur la Lune », 1969

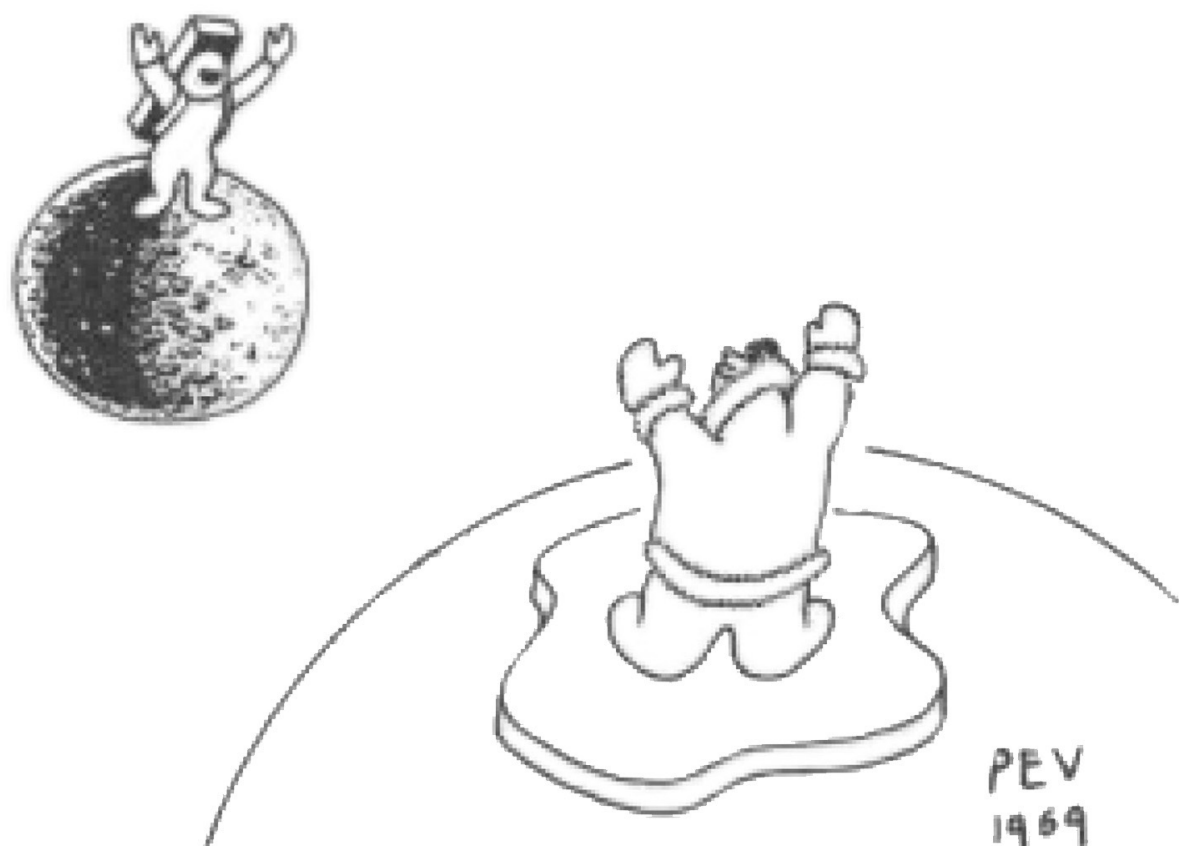

24. Günther Anders, Der Blick vom Mond, Reflexionen über Weltraumflüge, Munich, C.H. Beck, 1970, p. 12, traduit par l'auteur. 


\section{Antarctique et espace : fin et suite de la géographie}

\section{L'ANTARCtique, MARS Et LA LUNE}

Plusieurs champs d'études menées en Antarctique depuis l'Année géophysique internationale (1957-1958) ont connu des adaptations dans le domaine du spatial habité et ont servi à inspirer la recherche de la vie sur Mars. Deux domaines en ont particulièrement marqué le développement: les études d'isolation et de confinement extrême et les essais de praticabilité de différentes technologies spatiales.

L'envoi de scientifiques en Antarctique pour des périodes d'hiver, c'est-à-dire pour des périodes de plus de six mois sans lumière naturelle dans un espace confiné et en isolement extrême, a permis de récolter des données importantes sur le développement psychologique et physiologique de l'homme dans des environnements extrêmes. Deux disciplines importantes sont nées de cette alliance entre les sciences et les nouvelles géographies d'exploration d'après-guerre - la psychologie environnementale et la physiologie environnementale qui ont pour objet l'étude de l'homme dans des milieux montagneux, polaire, océanique et spatial. Les sciences spatiales ont particulièrement profité de ces données pour améliorer leurs méthodes de détermination des aptitudes du futur personnel pour des missions spatiales habitées. Après s'être d'abord intéressé au problème de l'adaptation de l'homme à l'absence de pesanteur, l'isolement extrême et le confinement ont été identifiés dès les années soixante par von Braun et d'autres chercheurs de la NASA comme des facteurs cruciaux dans le cadre d'une future mission lunaire.

Des recherches pionnières menées en écologie (principalement en microbiologie) à partir du milieu des années soixante et tout au long des années soixante-dix ont aussi identifié les sols des "Vallées sèches » de McMurdo en Antarctique (une région désertique, rocheuse et dénuée de neige et de glace) comme une approximation adéquate pour tester des technologies spatiales qui devraient servir à étudier la présence de vie sur le sol martien - même en reconnaissant que l'environnement en Antarctique diffère considérablement de celui qu'on trouve sur Mars. Horowitz, directeur de la section «bioscience » au Jet Propulsion Laboratory (JPL) et du California Laboratory of Technology (CalTech) et chefbiologiste du programme Viking, a envoyé à la fin des années soixante le microbiologiste Roy Cameron (JPL) en Antarctique. Il a passé huit saisons dans les vallées sèches étudiant la composition des sols pour trouver des indices des interactions du sol et des micro-organismes avec l'environnement et testant les instruments qui pourraient enfin aider, lors du programme Viking, à détecter la présence de traces de vie dans le sol martien - un espoir qui ne s'est pas confirmé au grand désespoir d'un bon nombre de scientifiques impliqués.

Cette tradition de tester des technologies en Antarctique avant l'envoi dans l'Espace s'est maintenue jusqu'à notre époque. La NASA, tout au long de son histoire, a renouvelé plusieurs fois ses ambitions polaires en signant de multiples contrats avec la National Science Foundation, le principal opérateur du programme scientifique américain en Antarctique. L'une des études récentes, exécutée entre 2008 et 2009 en Antarctique à la base McMurdo, consistait à tester l'utilité pratique d'un habitat gonflable en vue de futures missions lunaires ou martiennes habitées. L'habitat pressurisé, chauffé et relativement faible en poids a été testé en outre pour son isolation thermique et sa tolérance aux dommages dans des environnements extrêmes. 


\section{DROIT ESPACE ET ANTARCTIQUE}

\section{Principes du droit de l'espace}

- Le droit de l'espace a été mis en place dans les années 1960 via cinq grands traités élaborés par les Nations Unies constituant la Magna Carta du droit international de l'espace.

- Le principal est le Traité sur les principes régissant les activités des États en matière d'exploration et d'utilisation de l'espace extra atmosphérique, y compris la Lune et les autres corps célestes entré en vigueur le 10 octobre 1967.

- Il a été largement accepté par la communauté internationale

- Selon l'article I du traité de 1967, l'exploration et l'utilisation de l'espace extra atmosphérique, y compris la Lune et les autres corps célestes, doivent se faire pour le bien et dans l'intérêt de tous les pays, quel que soit le stade de leur développement économique ou scientifique ; elles sont l'apanage de l'humanité tout entière. L'espace extra atmosphérique, y compris la Lune et les autres corps célestes, peut être exploré et utilisé librement par tous les États sans aucune discrimination, dans des conditions d'égalité et conformément au droit international, toutes les régions des corps célestes devant être librement accessibles. Les recherches scientifiques sont libres dans l'espace extra atmosphérique, y compris la lune et les autres corps célestes, et les États doivent faciliter et encourager la coopération internationale dans ces recherches.

- Cet article 1 résume à lui seul l'esprit du droit de l'espace : une zone internationale où il faut prendre en compte les intérêts de tous les pays; une utilisation libre dans des conditions d'égalité ; un libre accès.

- L'espace extra atmosphérique, y compris la Lune et les autres corps célestes, ne peut faire l'objet d'appropriation nationale par proclamation de souveraineté, ni par voie d'utilisation ou d'occupation, ni par aucun autre moyen (art 2 du Traité de 1967).

- Il n'y a pas d'interdiction d'exploiter les ressources. L'Accord sur la Lune de 1979 précise que le jour où cette exploitation sera rendue possible, il sera nécessaire de mettre en place un régime international. Rien ne s'est produit de tel depuis 30 ans.

Le Droit de l'Antarctique, modifié récemment, va beaucoup plus loin quant à la protection des ressources.

\section{Principes du droit de l'Antarctique}

- Convention sur la protection des phoques de l'Antarctique de 1972 (entrée en vigueur en 1978)

- Convention sur la conservation de la faune et la flore marines de l'Antarctique de 1980 (entrée en vigueur en 1982)

- Convention pour la réglementation des activités sur les ressources minérales antarctiques de 1988. Cette convention n'est jamais entrée en vigueur en raison du refus par certains États de la ratifier : ils estiment que ce traité ne permet pas de concilier suffisamment exploitation des ressources minérales et protection de l'environnement. Elle a conduit à l'adoption du protocole de Madrid en 1991.

- Protocole au Traité sur l'Antarctique relatif à la protection de l'environnement en Antarctique de 1991 entré en vigueur en 1998 (ou protocole de Madrid) qui donne un volet environnemental au traité.

- Il désigne l'Antarctique comme une « réserve naturelle consacrée à la paix et à la science » (Article 2). 
- L'article 3 de cet instrument définit les principes fondamentaux qui s'appliquent aux activités humaines dans l'Antarctique.

- L'article 7 interdit toutes les activités relatives aux ressources minérales de l'Antarctique autres que la recherche scientifique. Jusqu'en 2048, il ne peut être modifié qu'avec l'accord unanime de toutes les Parties consultatives au Traité sur l'Antarctique.

- De surcroît, l'interdiction des activités relatives aux ressources minérales de l'Antarctique continue, à moins que ne soit en vigueur un régime juridique obligatoire concernant lesdites activités (article 25.5). 